\title{
Spectrum Utilization Efficiency of Elastic Optical Networks Utilizing Coarse Granular Routing
}

\author{
Hai-Chau Le and Ngoc T. Dang \\ Posts and Telecommunications Institute of Technology, Hanoi, Vietnam \\ Computer Communication Labs, The University of Aizu, Aizu-wakamatsu, Japan \\ E-mail: chaulh@ptit.edu.vn,ngocdt@ptit.edu.vn
}

Keywords: elastic optical network, optical cross-connect, spectrum selective switch, routing and spectrum assignment

Received: March 29, 2018

\begin{abstract}
In this paper, we have investigated an elastic optical network that uses coarse granular routing based on our recently developed coarse granular node architecture. The developed coarse granular optical cross-connect $(\mathrm{OXC})$ architecture that enables routing bandwidth-flexible lightpaths coarse-granularly is based on coarser granular selective spectrum switches. The network takes the advantages of both elastic optical networking and coarse granular routing technologies to cope with the trade-off between the link cost and the node cost in order to build a spectrum-and-cost efficient solution for future Internet backbone networks. We have evaluated the hardware scale requirement and the spectrum utilization efficiency of the network with typical modulation formats under various network and traffic conditions. We also compared the spectrum utilization of our network to that of corresponding traditional WDM network and conventional elastic optical network. Numerical results verified that, similar to conventional elastic optical network, the proposed network offers a substantial spectrum saving comparing to traditional WDM network.
\end{abstract}

Povzetek: Prispevek uvede izvirno elastično optično omrežje in analizira lastnosti kot učinkovitost.

\section{Introduction}

The ever-increasing Internet traffic growth has been continuously spurred by newly emerged highperformance and bandwidth-killer applications such as 4k/HD/ultra-HD video, e-Science and cloud/grid computing [1,2]. To cope with the explosive traffic increment and to support further mobility, flexibility and bandwidth heterogeneity, the necessity of cost-efficient and bandwidth-abundant flexible optical transport networks has become more and more critical [3, 4]. To scale up to Terabit/s, current optical transport networks based on current WDM technology with a fixed ITU-T frequency grid will encounter serious issues due to the stranded bandwidth provisioning, inefficient spectral utilization, and high cost [3]. Recent research efforts on optical transmission and networking technologies that are oriented forward more efficient, flexible, and scalable optical network solutions [4] can be categorized into two different approaches that are: 1) improving the link resource utilization/flexibility and 2) minimizing the node system scale/cost.

The first approach which aims to enhance the spectrum utilization and the network flexibility is currently dominated by the development of elastic optical networking technology [5-12]. Elastic optical networks (EON) realize spectrum- and energy-efficient optical transport infrastructure by exploiting bitrate-adaptive spectrum resource allocation with flexible spectrum/frequency grid and distance-adaptive modulation $[8,9]$. They are also capable of providing dynamic spectrum-effective and bandwidth-flexible end- to-end lightpath connections while offering Telcos (IT/communication service providers) the ability to scale their networks economically with the traffic growth and the heterogeneity of bandwidth requirement [10, 11]. However, EON is still facing challenges owing to the lack of architectures and technologies to efficiently support bursty traffic on flexible spectrum. It also requires more complicated switching systems and more sophisticated network planning and provisioning control schemes [12].

On the other hand, the second approach targets the development of cost-effective, scalable and large scale optical switching systems [13-18]. One of the most attractive direction is the use of coarse granular optical path (lightpath) switching [16-17] that can be realizable with optical/spectrum selective switching technologies [18]. Spectrum selective switches (SSSs) are available with multiple spectrum granularities which are defined as the number of switching spectrum bands. It is demonstrated that, with a common hardware technology (i.e. MEMS, PLC, LCoS, ...), the hardware scale is increased dramatically as finer granular SSSs are applied. Coarser granular SSSs are simpler and more costeffective but, their routing flexibility is limited more severely. Unfortunately, this routing limitation may seriously affect the network performance, especially in case of dynamic wavelength path provision. In other words, node hardware scale/cost reduction only can be attained at a cost of certain routing flexibility restriction. Hence, it is desirable to enhance the node routing 
flexibility while still keeping the hardware reduction as large as possible.

Based on that, in order to exploit elastic optical networking and coarse granular switching for a realizing cost-efficient, spectrum effective and flexible optical transport network, we have recently developed a singlelayer optical cross-connect architecture based on coarse granular switching spectrum selective switches. Elastic optical network that employs the developed OXC architecture is still capable of exploiting elastic optical networking technology while attaining a substantial hardware reduction. We have also evaluated the network spectrum utilization in various network scenarios such as single modulation format (BPSK, QPSK, 8QAM and 16QAM) and distance-adaptive scheme. Numerical evaluations verified that, like a conventional elastic optical network, the proposed network can obtain a significant spectrum saving comparing to the corresponding traditional WDM network. A preliminary version of this work with the proposal and limited basic numerical effectiveness evaluation of a bandwidthflexible and coarse granular optical cross-connect architecture was presented at the SoICT conference [19].

\section{Elastic optical network utilizing coarse granular routing}

\subsection{Developed coarse granular routing OXC architecture [19]}

Most existing optical cross-connect systems are realized by optical selective switch technology which is one of the most popular and mature optical switching technologies. For constructing a high-port count OXC, multiple spectrum selective switches can be cascaded to create a higher port count SSS to overcome the limitation of commercially available SSS port count which is currently $20+$ and unlikely will be substantially enhanced cost-effectively in the near future [4, 18]. Therefore, larger scale optical cross-connect system requires more and/or higher port count SSSs. Moreover, spectrum selective switches are still costly and complicated devices. SSS cost/complexity strongly relies on the number of switching spectrum bands per fiber (also called the spectrum granularity). Finer granular SSSs are more complicated as well as have greater hardware scale and consequently, become more expensive.

Based on that observation, in order to exploit elastic optical network technology while keeping the hardware scale reasonably small, we have recently developed a coarse granular routing elastic optical cross-connect architecture (denoted as GRE network) for realizing flexible bandwidth large scale optical transport networks [19]. Figure 1 shows the developed OXC system in which, instead of using fine granular SSSs in traditional bandwidth-variable OXC in elastic optical networks, coarse granular spectrum selective switches are implemented to build a cost-efficient high-port count OXC system. Unlike neither traditional WDM networks that divide the spectrum into individual channels with the

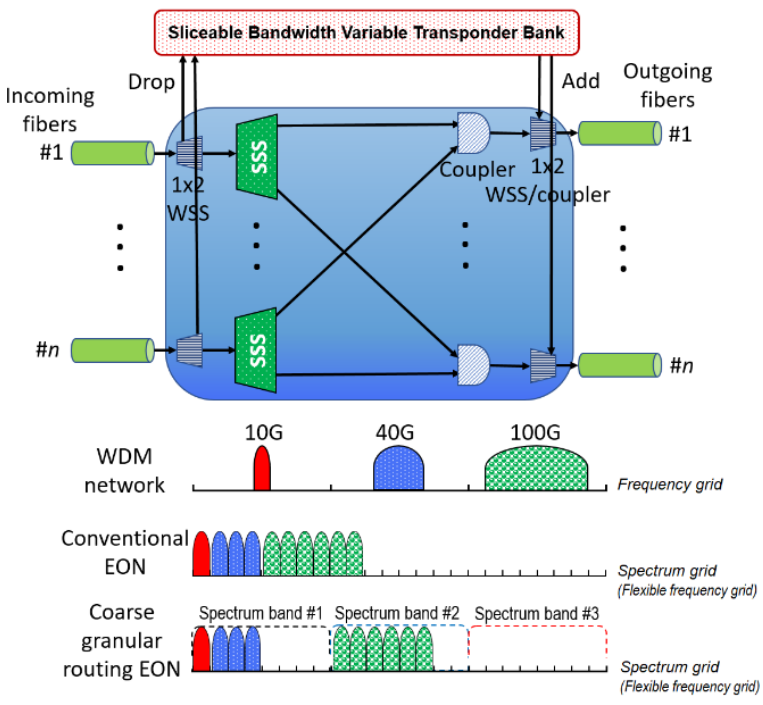

Figure 1: Coarse granular routing $\mathrm{OXC}$ architecture [19].

fixed channel spacing of either $50 \mathrm{GHz}$ or $100 \mathrm{GHz}$ specified by ITU-T standards nor elastic optical networks that employ a flexible frequency grid with a fine granularity (i.e. $12.5 \mathrm{GHz}$ ), the developed coarse granular routing elastic optical network employs the same flexible frequency grid but it routes lightpaths at the spectrum band level, so called "coarse" granular routing entity - GRE, through coarse granular OXCs; all spectrum slots of a band must be routed together as a single entity.

\subsection{Hardware scale requirement}

Practically, the cost and the control complexity of WSS/SSS-based systems depend strongly on the switch scale. Hence, switch scale minimization plays a key role for creating cost-effective large-scale WSS/SSS-based OXCs. Among recently available commercial optical switch technologies for constructing wavelength selective switch and/or spectrum selective switch systems, MEMS-based system are known as one of the most popular and widely adopted technology. Hence, to estimate the effectiveness of our recently developed OXC architecture, for simplicity, we just consider MEMS-based spectrum selective switches whose hardware scale mainly depends on the number of necessary elemental MEMS mirrors. Furthermore, without the loss of generality, adding/ dropping portions which can be simple $1 \times 2$ SSSs or couplers are also neglected. The switch scale of OXC systems, consequently, is quantified by the total MEMS mirrors required by SSS components.

We assume that the transmission bandwidth of a fiber is $C_{\text {fiber }}$, the channel spacing based on ITU-T frequency grid of traditional WDM network is $G_{W D M}$ $\left(G_{W D M}=100 \mathrm{GHz}\right)$ and EON channel spacing is $G_{E O N}$ $\left(G_{E O N} \ll G_{W D M}\right)$. The number of wavelengths per fiber, $W_{W D M}$, in WDM network can be calculated as,

$$
W_{W D M}=\frac{C_{\text {fiber }}}{G_{W D M}}
$$


while the number of spectrum slots per fiber, $S$, of elastic optical network is given by,

$$
S=\frac{C_{\text {fiber }}}{G_{E O N}}
$$

Let $\mathrm{W}$ denote the size of coarse granular routing entity (i.e. GRE granularity), the number of spectrum slots per GRE, and let $\mathrm{S}$ be the total number of spectrum slots that can be accommodated in a fiber; $1 \leq W \leq S$ and $S$ is divisible by $W$ and we have $L=S / W(1 \leq L \leq S)$ is the number of switching spectrum slots per fiber. Each mirror of a MEMS-based selective spectrum switch is dedicated to a spectrum slot (or spectrum band) and so, each SSS needs $L$ MEMS mirrors. Note that all spectrum slots of a GRE are simultaneously switched by a mirror. Hence, total number of MEMS mirrors required in WDM OXC, elastic OXC and the proposed GRE architecture are calculated as following,

$$
\begin{aligned}
M_{W D M} & =n W_{W D M}\left(1+\left\lceil\frac{n-1}{M}\right\rceil\right) \\
M_{E O N} & =n S\left(1+\left\lceil\frac{n-1}{M}\right\rceil\right) \\
M_{G R E} & =n L\left(1+\left\lceil\frac{n-1}{M}\right\rceil\right)
\end{aligned}
$$

where $n$ is the input/output fiber number $(n>0), M$ is the maximal selective switch size (i.e. port count) and $L$ is the GRE granularity. Table 1 summarizes the switch scale calculating formulas. The formulations also imply that the total number of necessary mirrors of an SSS is decreased as the applied GRE granularity becomes

\begin{tabular}{|c|c|c|c|}
\hline \multirow{2}{*}{$\begin{array}{c}\text { OXC } \\
\text { Architecture }\end{array}$} & \multicolumn{2}{|c|}{ Switching component } & \multirow{2}{*}{$\begin{array}{c}\text { Switch scale } \\
\text { (Total mirror number) }\end{array}$} \\
\hline & $\begin{array}{l}\text { Switch } \\
\text { element }\end{array}$ & Total number & \\
\hline $\begin{array}{c}\text { Conventional } \\
\text { WDM }\end{array}$ & WSS & \multirow{3}{*}{$n\left(1+\left\lceil\frac{n-1}{M}\right\rceil\right)$} & $n W_{W D M}\left(1+\left[\frac{n-1}{M}\right\rceil\right)$ \\
\hline Developed $O X C$ & $\begin{array}{c}\text { Coarser } \\
\text { granular SSS }\end{array}$ & & $n \frac{S}{W}\left(1+\left\lceil\frac{n-1}{M}\right\rceil\right)$ \\
\hline Elastic OXC & SSS & & $n S\left(1+\left\lceil\frac{n-1}{M}\right\rceil\right)$ \\
\hline
\end{tabular}
greater or it means that applying coarser granular SSSs (SSSs with greater $W$ ) will help to reduce the switch scale of OXC systems.

Table 1: Switch scale calculation.

Figure 2 shows the switch scale requirement of the developed OXC architecture, in terms of MEMS mirrors, with respect to both the number of input/output fibers (the port count) and the number of switching spectrum bands per fiber. The graph demonstrates that the hardware scale increases as the number of input fibers becomes greater. The hardware scale increment becomes much more significant if more number of switching bands per fiber (finer GRE granularity) is applied. Hence, a great deal of hardware scale/cost reduction can be achieved if the GRE granularity is limited at a reasonable value. It implies that coarse granular routing elastic optical network (using coarse granular SSSs) can be considered as a promising solution for creating costeffective and bandwidth-abundant transport networks.

Moreover, Figure 3 shows the hardware scale comparison of the three comparative OXC architectures that are traditional $\mathrm{OXC}$, elastic $\mathrm{OXC}$ and coarse

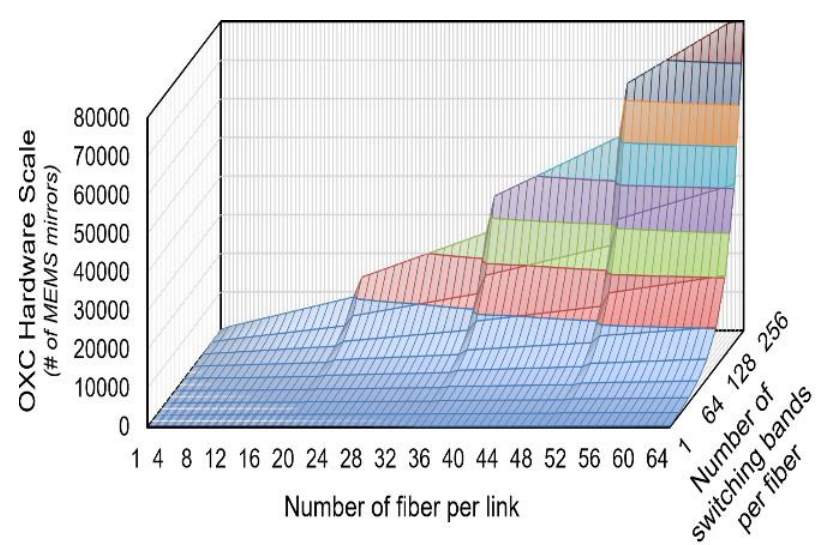

Figure 2: Hardware scale requirement of spectrum selective switch-based OXC.

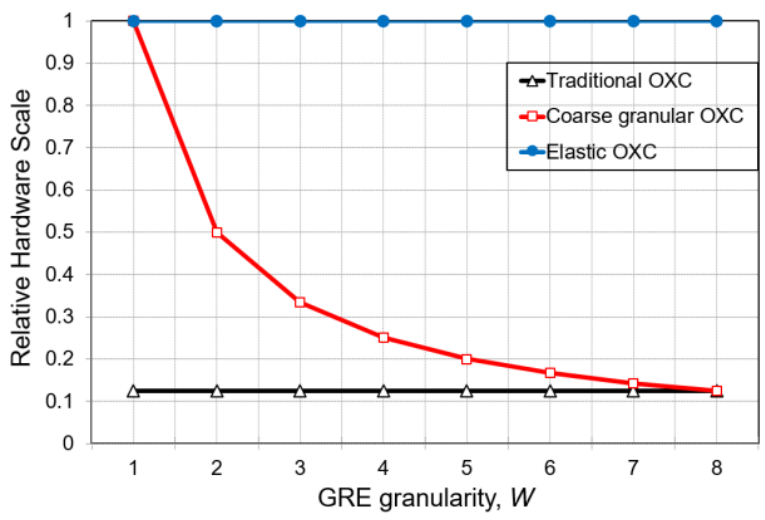

Figure 3: Hardware scale comparison.

granular OXC when the WDM channel spacing is 100 $\mathrm{GHz}$ and the spectrum slots of EON is $12.5 \mathrm{GHz}$. Due to the use of large channel spacing, i.e. $100 \mathrm{GHz}$ or 50 $\mathrm{GHz}$, traditional OXC needs the smallest hardware scale. On the other hand, thanks to the reduction of the number of switching spectrum bands, coarse granular OXC needs fewer number of switching elements comparing to conventional elastic optical cross-connect. Obviously, the hardware scale reduction offered by coarse granular OXC is enhanced, especially when coarser granular routing is applied (greater GRE granularity).

\subsection{Network routing operation}

Unlike conventional OXCs in WDM or EON networks, the developed GRE node suffers from a intra-node routing limitation due to the use of coarser granular spectrum selective. Figure 4 illustrates the routing principle of the developed coarse granular routing optical cross-connect architecture. In elastic optical network which uses the developed coarse granular routing node architecture (so called coarse granular routing elastic optical network), lightpaths of a spectrum band can be added/dropped flexibly and dynamically by $1 \times 2$ SSSs/optical coupler equipped for incoming and outgoing fibers and sliceable bandwidth variable transponders with the spectrum band capacity. Different from conventional elastic optical networks in which spectrum slots of each lightpath can be routed separately, in this network, whole spectrum slots of a spectrum band 
from an incoming fiber must be switched together as one entity due to the coarse granular routing restriction of spectrum selective switches. It means that all lightpaths which are assigned to spectrum slots of the same spectrum band have to be routed to a common output fiber. This restriction imposed by the spectrum band granularity of SSSs limits the routing flexibility of the proposed OXC architecture. The node routing flexibility depends on the SSS spectrum granularity. In coarse granular routing elastic optical network, finer SSS granularity can be applied to improve the node routing flexibility, however, utilizing finer granular SSSs may cause a rapid increase in hardware-scale/cost. Hence, the SSS granularity must be carefully determined while considering the balance the node routing flexibility against the hardware scale/cost.

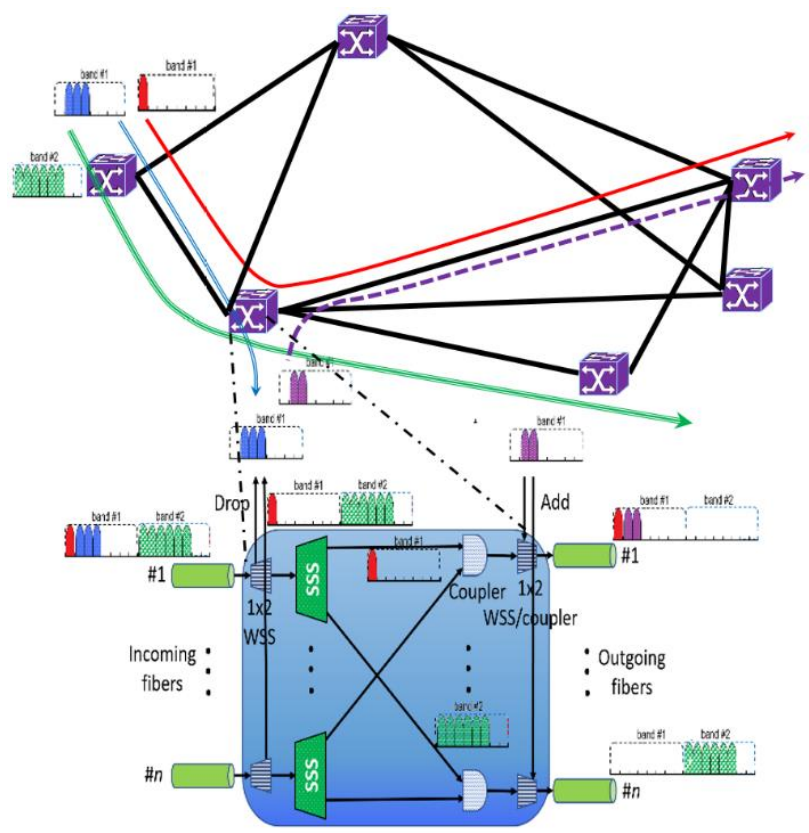

Figure 4: Coarse granular routing principle.

On the other hand, similar to conventional elastic optical networks, the coarse granular routing elastic optical network also can support single or multiple modulation formats flexibly and dynamically. Each lightpath can be assigned to a pre-determined modulation format (single modulation format scenario) or an appropriate modulation format according to its distance (called distance-adaptive scenario). In distance-adaptive scheme, for a given traffic capacity, modulating optical signal with a higher-order format offers higher capacity per spectrum slot and consequently, requires less number of spectrum slots. It means that applying higher-order modulation format obtains higher spectrum efficiency but its optical transparent reach is shortened and consequently, more frequent regeneration and/or more regeneration resources are required. Inversely, utilizing lower-order modulation formats might lower the spectrum slot capacity and hence, may cause an increment in the required spectrum slot number. Hence, impact of the modulation format assignment scenarios on the network spectrum utilization needs to be clarified.

\section{Spectrum usage evaluation}

\subsection{Theoretical spectrum utilization analysis}

In this section, we evaluate the spectrum utilization of three comparative optical networks including WDM, traditional EON and coarse granular routing elastic optical networks. Without the loss of generality, we assumed the following parameters. The channel spacing based on ITU-T frequency grid of traditional WDM network is $100 \mathrm{GHz}\left(G_{W D M}=100 \mathrm{GHz}\right.$, the most popular frequency grid) and the lowest order modulation format (i.e. BPSK) is applied. Elastic optical network is deployed with a typical channel spacing of $12.5 \mathrm{GHz}$ $\left(G_{E O N}=12.5 \mathrm{GHz}\right)$ and five modulation format assignment scenarios including four single modulation format (BPSK, QPSK, 8QAM and 16QAM) and a distance-adaptive schemes.

\section{1) Point-to-point link}

In this part, we simply estimated the spectrum utilization of a single point-to-point link with 3 comparative technologies including WDM, EON and our coarse granular routing EON (denoted as GRE). We assumed that the considered link includes Hs,d hops and has the total distance of $D_{s, d}$ where $(s, d)$ is the source and destination node pair of the link, and requested bitrate of the connection on the link is $R_{s, d}$ (Gbps).

Based on that, let $C_{W D M}$ be the channel capacity of BPSK WDM, the number of spectrum slots needed in the conventional WDM network, $N S_{W D M}(s, d)$, can be calculated as,

$$
N S_{W D M}(s, d)=\left\lceil\frac{R_{s, d}}{C_{W D M}}\right\rceil H_{s, d}
$$

Hence, the total WDM spectrum is,

$$
S_{W D M}(s, d)=G_{W D M}\left[\frac{R_{s d}}{C_{W D M}}\right] H_{s, d} .
$$

For conventional elastic optical network, the spectrum slot number required in a single modulation format scheme (which uses only one modulation format of optical signals) is given by,

$$
N S_{E O N-M O D}(s, d)=\left\lceil\frac{R_{S, d}}{C_{E O N-M O D}}\right\rceil H_{s, d}
$$

where, $M O D$ denotes the selected modulation format (it will be replaced by BPSK, QPSK, 8QAM or 16QAM) and $C_{E O N-M O D}$ is the corresponding slot capacity. From Equation (8), the necessary spectrum of single modulation format elastic optical link can be evaluated as,

$$
S_{E O N-M O D}(s, d)=G_{E O N}\left\lceil\frac{R_{s, d}}{C_{E O N-M O D}}\right\rceil H_{s, d} .
$$

Let $\alpha$ be the spectrum grooming ratio $(0<\alpha \leq 1)$; $\alpha=\frac{x}{G R E}$ where GRE is the GRE granularity, the capacity of coarse granular routing entity, and $x$ is the average number of spectrum slots which carry the traffic in a coarse granular routing entity. Consequently, the number 
of spectrum slots and the corresponding total spectrum required for coarse granular routing EON link are respectively calculated as,

$$
N S_{G R E-M O D}(s, d)=\frac{1}{\alpha}\left[\frac{R_{s, d}}{G R E \times C_{E O N-M O D}}\right] H_{s, d},
$$

and,

$$
S_{G R E-M O D}(s, d)=\frac{G R E \times G_{E O N}}{\alpha}\left\lceil\frac{R_{S, d}}{G R E \times C_{E O N-M O D}}\right\rceil H_{s, d} .
$$

On the other hand, for the distance-adaptive scheme of both conventional EON and our GRE networks, the modulation format of each lightpath is determined individually and assigned dynamically according to total distance of the lightpath. Therefore, if we assume that the simplest modulation format assignment strategy, which assigns the possible highest order of modulation format, is used, the total spectrum slot number required by the distance adaptive scheme of EON and coarse granular routing EON networks are,

$$
\begin{aligned}
& N S_{E O N-\text { adap }}(s, d)= \\
& \left\{\begin{array}{lc}
{\left[\frac{R_{S, d}}{C_{E O N-16 Q A M}}\right] H_{S, d}} & \text { if } D_{S, d} \leq L_{16 Q A M} \\
{\left[\frac{R_{S, d}}{C_{E O N-8 Q A M}}\right] H_{S, d}} & \text { if } L_{16 Q A M}<D_{S, d} \leq L_{8 Q A M} \\
\left\lceil\frac{R_{S, d}}{C_{E O N-Q P S K}}\right] H_{S, d} & \text { if } L_{8 Q A M}<D_{S, d} \leq L_{Q P S K} \\
\left\lceil\frac{R_{S, d}}{C_{E O N-B P S K}}\right] H_{S, d} & \text { otherwise, }
\end{array}\right.
\end{aligned}
$$

and,

$$
\begin{gathered}
N S_{G R E-a d a p}(s, d)= \\
\left\{\begin{array}{lr}
\frac{1}{\alpha}\left[\frac{R_{S, d}}{G R E \times C_{E O N-16 Q A M}}\right] H_{S, d} & \text { if } D_{S, d} \leq L_{16 Q A M} \\
\frac{1}{\alpha}\left[\frac{R_{s, d}}{G R E \times C_{E O N-8 Q A M}}\right] H_{S, d} & \text { if } L_{16 Q A M}<D_{S, d} \leq L_{8 Q A M} \\
\frac{1}{\alpha}\left[\frac{R_{S, d}}{G R E \times C_{E O N-Q P S K}}\right] H_{S, d} & \text { if } L_{8 Q A M}<D_{S, d} \leq L_{Q P S K} \\
\frac{1}{\alpha}\left[\frac{R_{S, d}}{G R E \times C_{E O N-B P S K}}\right] H_{S, d} & \text { otherwise. }
\end{array}\right.
\end{gathered}
$$

From Equations (12) and (13), the required spectrum utilization of elastic optical link and that of coarse granular routing EON are estimated accordingly by,

$$
S_{E O N-\text { adap }}(s, d)=G_{E O N} N S_{E O N-\text { adap }}(s, d)
$$

and,

$$
S_{\text {GRE-adap }}(s, d)=G R E \times G_{E O N} N S_{G R E-a d a p}(s, d) .
$$

\section{2) Spectrum utilization of the network}

Given a network topology $G=\{V, E\}$ in which $V$ is the set of nodes, $|V|=n$, and $E$ is set of links. For each node pair $(s, d)((s, d) \in V \mathrm{x} V)$, we assume that the traffic load requested from the source node, $s$, to the destination node, $d$, is $R_{s, d}$, the hop count and the distance of the route connecting $s$ and $d$ are $H_{s, d}$ and $D_{s, d}$ respectively.

Based on the calculations given in Equations (7) and (9), total spectrum required in conventional WDM network is,

$$
S_{W D M}=\sum_{\substack{(s, d) \in V \times V \\ s \neq d}} G_{W D M}\left\lceil\frac{R_{s, d}}{C_{W D M}}\right\rceil H_{s, d},
$$

and the spectrum utilization of elastic optical networks for single modulation format scheme is given by,

$$
S_{E O N-M O D}=\sum_{\substack{(s, d) \in V \mathrm{x} V \\ s \neq d}} G_{E O N}\left\lceil\frac{R_{s, d}}{C_{E O N-M O D}}\right\rceil H_{S, d} .
$$

Similarly, from Equation (11), we have the total spectrum utilization of coarse granular routing elastic optical network for single modulation format scheme as following,

$S_{G R E-M O D}=\sum_{\substack{(s, d) \in V \mathrm{x} V \\ s \neq d}} \frac{G R E \times G_{E O N}}{\alpha}\left\lceil\frac{R_{s, d}}{G R E \times C_{E O N-M O D}}\right\rceil H_{s, d}$.

Moreover, in distance-adaptive scheme, elastic optical networks including both conventional network and our developed network are able to assign modulation format dynamically. In fact, there are many different modulation assignment strategies, i.e. shortest path first (or least spectrum), least generating resource,... Depending on the applied strategy, the implementing portions of available modulation formats can be varied. If we assume that $\alpha, \beta, \gamma$ and $\delta$ are coefficients which determine the distribution of the selected modulation formats (BPSK, QPSK, 8QAM and 16QAM) in the network respectively, $\quad \alpha \geq 0, \quad \beta \geq 0, \quad \gamma \geq 0, \quad \delta \geq 0 \quad$ and $\alpha+\beta+\gamma+\delta=1$. Based on Equations (17) and (18), the required spectrum of distance-adaptive conventional elastic optical network and that of coarse granular routing EON network can be calculated as,

$$
\begin{gathered}
S_{E O N-\text { adap }}=\alpha S_{E O N-B P S K}+\beta S_{E O N-Q P S K} \\
+\gamma S_{E O N-8 Q A M}+\delta S_{E O N-16 Q A M} \\
S_{\text {GRE-adap }}=\alpha S_{G R E-B P S K}+\beta S_{G R E-Q P S K} \\
+\gamma S_{G R E-8 Q A M}+\delta S_{G R E-16 Q A M}
\end{gathered}
$$

This means that the performance of distance adaptive networks is in the middle comparing to other single modulation format elastic networks.

From Equations (16)-(20), the length of lightpaths, in term of both hop count and distance, significantly affects the usage of spectrum; longer paths are, more spectrum is required. It should be minimized to optimize the resource usage in elastic optical networks. In other words, the shortest paths should be used for lightpaths. However, note that implementing the shortest paths simply may result in a substantial spectrum collision.

\subsection{Numerical results and discussion}

To estimate the performance efficiency of the developed coarse granular routing elastic optical network, we utilized the following parameters for numerical evaluations. The frequency grid of WDM network is 100 $\mathrm{GHz}$ and the spectrum slot bandwidth of EON and GRE networks is $12.5 \mathrm{GHz}$. Two tested network topologies are pan-European optical transport network, COST266, and US backbone network, USNET (see Figure 5). Traffic load is represented by the total traffic demand requested between node pairs which is assigned randomly according to a uniform distribution in the range from 50 Gbps to $500 \mathrm{Gbps}$ (for each traffic load, 100 samples were tested and the average values were then plotted).

In the numerical experiments, we have also assumed comparative elastic optical networks provide four typical 
modulation formats that are BPSK, QPSK, 8QAM and 16QAM. Consequently, there are five experimental network scenarios that are four single modulation format schemes (BPSK, QPSK, 8QAM, and 16QAM) and a distance-adaptive scheme. The coarse granular switching group capacity, GRE (the number of spectrum slots per group), is set as a variable. In fact, we tested the GRE granularity of 2, 4, and 8 (in case GRE=1, GRE network is equivalent to conventional EON). The obtained results of the corresponding WDM network are used as a benchmark (its graph is always 1); all obtained results for EON and GRE networks are compared to that of the corresponding WDM network and the relative data will be demonstrated.

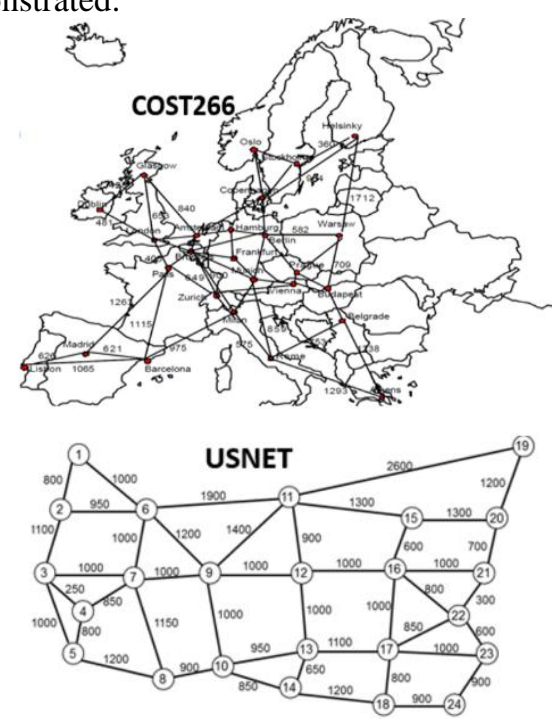

Figure 5: Tested network topologies.

Firstly, the spectrum usage comparison in the case of distance-adaptive scheme for the three comparative networks in COST266 and USNET topologies is illustrated in Figure 6 . The obtained results show that both the GRE network and conventional elastic optical network offer a significant spectrum saving comparing to the traditional WDM network; up to $65 \%$ (45\%) spectrum reduction can be achieved for COST266 (USNET) network topology with the traffic of $500 \mathrm{Gbps}$, thanks to the deployment of the flexible grid and dynamic modulation format assignment. It also demonstrates that the relative spectrum utilization of EON and GRE networks tends to decreased slightly as the traffic load becomes greater or finer granular routing is applied (smaller GRE granularity). That is because large traffic load can fill up huge channel spacing as used in conventional WDM networks and thus, using finer frequency grid does not help much to reduce the spectrum utilization. Note that, in this distance adaptive scheme, the spectrum utilization savings are less than those for 16QAM single modulation format scheme due to the possibility of implementing lower order modulation format to cope with the distance of required traffic without using any regenerating resource.

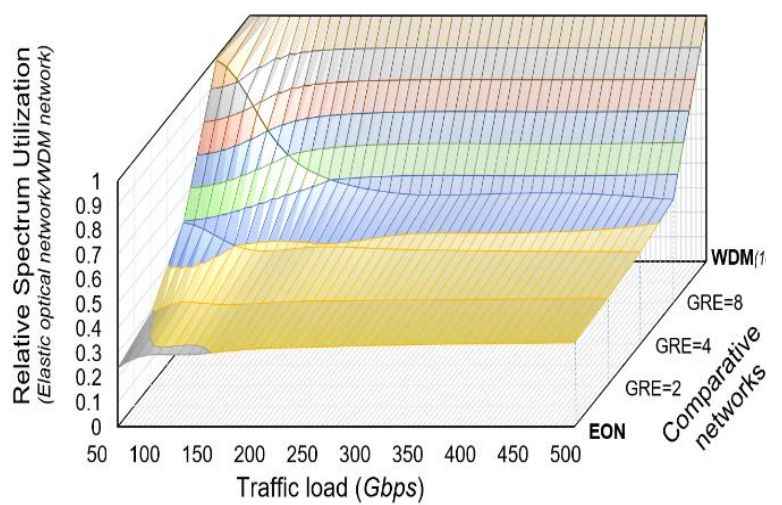

a) $\operatorname{COST} 266$

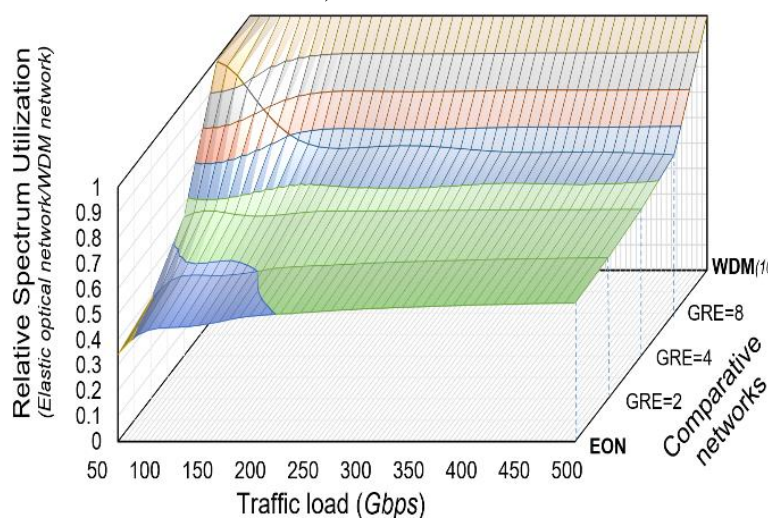

b) USNET

Figure 6: Spectrum utilization comparison for distance-adaptive scheme.

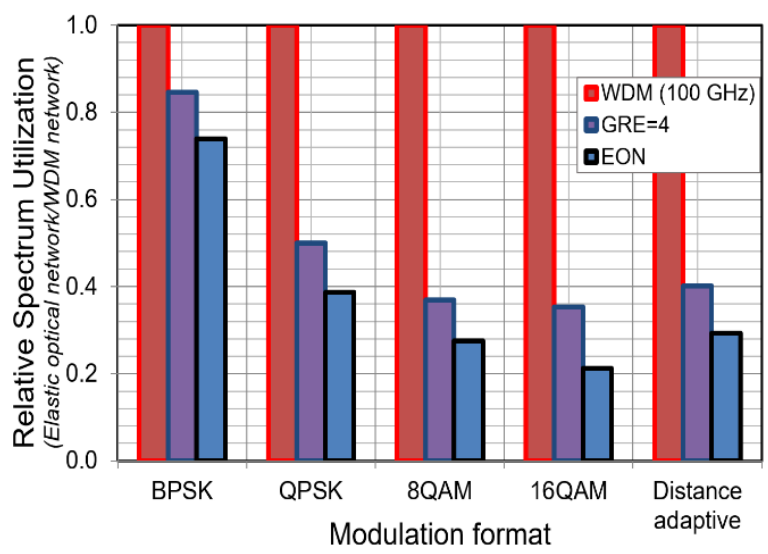

a) $\operatorname{COST} 266$

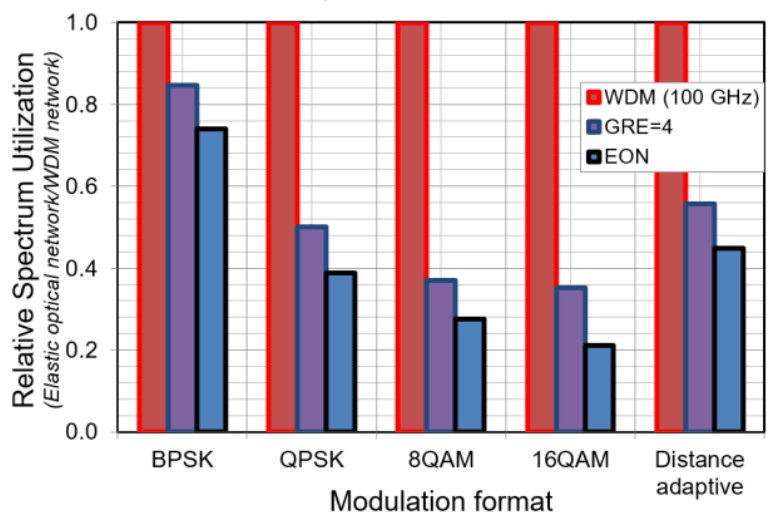

b) USNET

Figure 7: Impact of modulation formats. 
Moreover, to verify the impact of the flexible modulation format assignment on the network performance, we compared five different network scenarios including four single modulation format schemes (BPSK, QPSK, 8QAM and 16QAM) and distance-adaptive scheme with the traffic load of 100 Gbps. The comparative results are described in Figure 7. It is demonstrated that employing higher order modulation formats offers better spectrum saving. Even the developed GRE network can reduce the hardware scale, the spectrum utilization of our network (as $\mathrm{GRE}=4$ ) is more than that of EON due to the routing flexibility limitation. This also implies the importance of flexible modulation format assignment in saving spectrum while dealing the trade-off between the node routing flexibility (node cost) and the link resource requirement.

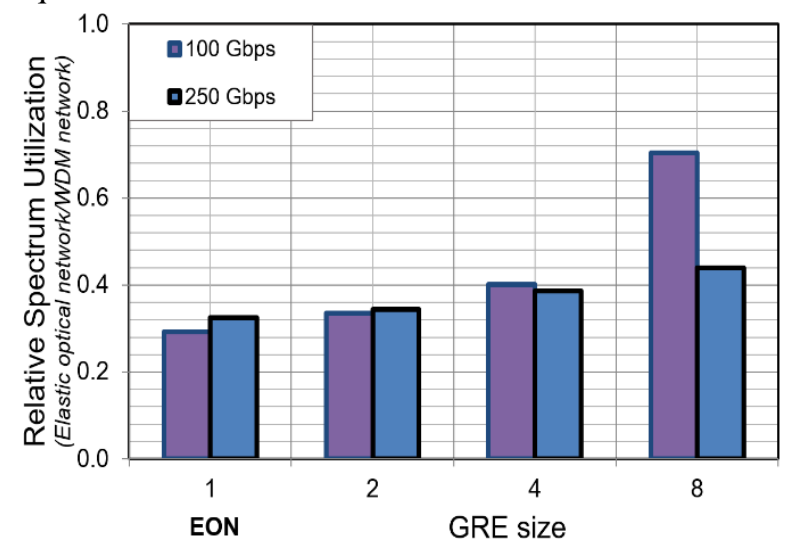

a) $\operatorname{COST} 266$

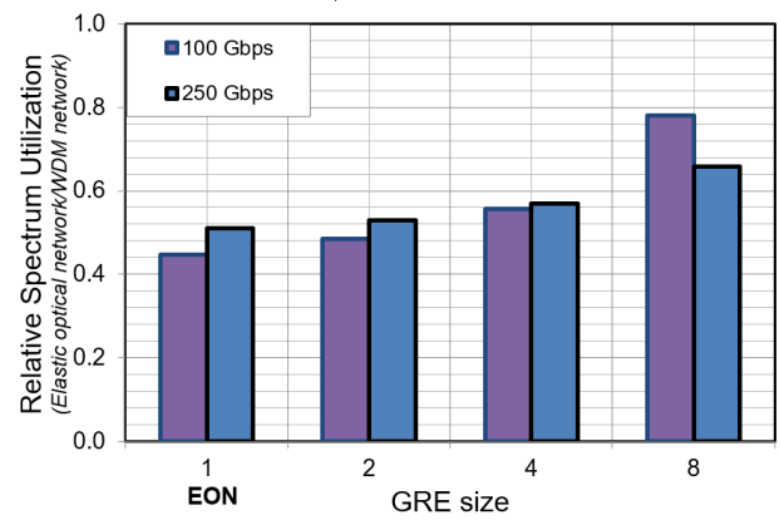

b) USNET

Figure 8: Dependence of the network spectrum usage on the GRE granularity.

Finally, Figure 8 shows the dependence of spectrum utilization on the GRE granularity applied when the traffic load is fixed at $100 \mathrm{Gbps}$ and $250 \mathrm{Gbps}$. Again, it is shown that finer granular routing (smaller GRE granularity) offers better network performance, in terms of spectrum utilization, especially for small traffic load. The reason is that small traffic load may not fill up whole the spectrum band switched in the GRE network. Finer granular routing is expected to reduce the spectrum utilization, however, it may result in an explosive increase in the hardware scale. Hence, in the network point of view, it is desirable to balance the spectrum usage and the hardware scale requirements.

\section{Conclusion}

We have introduced a coarse granular routing elastic optical network that employs the developed coarse granular spectrum selective switch-based optical crossconnect architecture. By imposing coarse granular spectrum selective switching, the developed network is still able to exploit elastic optical networking technology while attaining a significant hardware reduction. To evaluate the performance of the coarse granular routing elastic optical network, we have clarified its spectrum utilization in various network scenarios, single modulation format (including BPSK, QPSK, 8QAM and 16QAM) and distance adaptive schemes, under different traffic conditions. We also compared the spectrum utilization of the network to that of corresponding traditional WDM network and conventional elastic optical network. Numerical results verified that, similar to conventional elastic optical network, the proposed network offers a substantial spectrum saving, says up to $65 \%$, comparing to traditional WDM network. The developed network provides a promising solution to deal with the trade-off between node cost and link cost for creating cost-effective and spectrum-efficient future Internet backbone networks.

\section{Acknowledgment}

This research is funded by Vietnam National Foundation for Science and Technology Development (NAFOSTED) under grant number 102.02-2015.39.

\section{References}

[1] Cisco Visual Networking Index: Forecast and Methodology, Cisco system, 2014-2019. Retrieved from http://www.cisco.com/c/en/us/solutions/ collateral/service-provider/ip-ngn-ip-nextgeneration-network/white_paper_c11-481360.pdf

[2] E. B. Desurvire (2006). Capacity demand and technology challenges for lightwave systems in the next two decades. Journal of Lightwave Technology, IEEE, vol. 24, No. 12, pp. 4697-4710. https://doi.org/10.1109/JLT.2006.885772

[3] J. Berthold, A. Saleh, L. Blair, J. Simmons (2008). Optical networking: Past, present, and future. Journal of Lightwave Technology, IEEE, vol. 26, No. 9, pp. 1104-1118. https://doi.org/10.1109/JLT.2008.923609

[4] K. Sato, H. Hasegawa (2009). Optical Networking Technologies That Will Create Future BandwidthAbundant Networks. Journal of Optical Communications and Networking, IEEE/OSA, vol. 1, no. 2, pp. A81-A93. https://doi.org/10.1364/JOCN.1.000A81

[5] A. Jukan and J. Mambretti (2012). Evolution of Optical Networking Toward Rich Digital Media Services. Proceedings of the IEEE, IEEE, vol. 100, no. 4 , pp. $855-871$. 
https://doi.org/10.1109/JPROC.2011.2182076

[6] G. Bosco, V. Curri, A. Carena, P. Poggiolini, and F. Forghieri (2011). On the performance of NyquistWDM terabit superchannels based on PM-BPSK, PM-QPSK, PM-8QAM or PM-16QAM subcarriers. Journal of Lightwave Technology, IEEE, vol. 29, No.1, pp. 53-61.

https://doi.org/10.1109/JLT.2010.2091254

[7] G. Zhang, M. De Leenheer, A. Morea and B. Mukherjee (2013). A Survey on OFDM-Based Elastic Core Optical Networking. IEEE Communications Surveys \& Tutorials, IEEE, vol. 15 , no. 1 , pp. $65-87$. https://doi.org/10.1109/SURV.2012.010912.00123

[8] M. Jinno, H. Takara, B. Kozicki, Y. Tsukishima, Y. Sone, and S. Matsuoka (2009). Spectrum-Efficient and Scalable Elastic Optical Path Network: Architecture, Benefits, and Enabling Technologies. IEEE Communications Magazine, IEEE, vol. 47, pp. 66-73. https://doi.org/10.1109/MCOM.2009.5307468

[9] O. Gerstel, M. Jinno, A. Lord and S. J. B. Yoo (2012). Elastic optical networking: a new dawn for the optical layer?.IEEE Communications Magazine, IEEE, vol. 50, no. 2, pp. s12-s20. https://doi.org/10.1109/MCOM.2012.6146481

[10] A. Lord, P. Wright and A. Mitra (2015). Core Networks in the Flexgrid Era. Journal of Lightwave Technology, IEEE, vol. 33, no. 5, pp.1126-1135. https://doi.org/10.1109/JLT.2015.2396685

[11] M. Jinno, B. Kozicki, H. Takara, A. Watanabe, Y. Sone, T. Tanaka and A. Hirano (2010). Distanceadaptive spectrum resource allocation in spectrumsliced elastic optical path network. IEEE Communications Magazine, IEEE, vol. 48, no. 8, pp.138-145. https://doi.org/10.1109/MCOM.2010.5534599

[12] B. Chatterjee, N. Sarma and E. Oki (2015). Routing and Spectrum Allocation in Elastic Optical Networks: A Tutorial. IEEE Communications Surveys \& Tutorials, IEEE, vol. PP, no. 99, pp. 1. https://doi.org/10.1109/COMST.2015.2431731

[13] T. Zami, D. Chiaroni (2012). Low contention and high resilience to partial failure for colorless and directionless OXC. Proceedings of Photonics in Switching, OSA, paper Fr-S25-O15. Retrieved from https://ieeexplore.ieee.org/document/6608250

[14] I. Kim, P. Palacharla, X. Wang, D. Bihon, M. D. Feuer, S. L. Woodward (2012). Performance of Colorless, Non-directional ROADMs with Modular Client-side Fiber Cross-connects. Proceedings of Optical Fiber Communication Conference (OFC2012), OSA, paper NM3F.7. https://doi.org/10.1364/NFOEC.2012.NM3F.7

[15] Y. Li, L. Gao, G. Shen, L. Peng (2012). Impact of ROADM colorless, directionless and contentionless (CDC) features on optical network performance. Journal of Optical Communication and Networking, IEEE, vol. 4, No. 11, pp. B58-B67. https://doi.org/10.1364/JOCN.4.000B58
[16] H.-C. Le, H. Hasegawa, K. Sato (2014). Performance evaluation of large-scale multi-stage hetero-granular optical cross-connects. Optics Express, OSA, vol. 22, no. 3, pp. 3157-3168. https://doi.org/10.1364/OE.22.003157

[17] Y. Taniguchi, Y. Yamada, H. Hasegawa, and K. Sato (2012). A novel optical networking scheme utilizing coarse granular optical routing and fine granular add/drop. Proceedings of OFC/NFOEC, OSA, pp. JW2A.2.

https://doi.org/10.1364/NFOEC.2012.JW2A.2

[18] R. Hirako, K. Ishii, H. Hasegawa, K. Sato, H. Takahashi, M. Okuno (2011). Development of Single PLC-Chip Waveband Selective Switch that Has Extra Ports for Grooming and Termination. Proceedings of the 16th Opto-Electronics and Communications Conference, IEEE, pp. 492-493. Retrieved from https://ieeexplore.ieee.org/document/6015223

[19] Hai-Chau Le, Thanh Long Mai, Ngoc T. Dang (2017). Spectrum Utilization of Coarse Granular Routing Elastic Optical Networks. Proceedings of SoICT'17: Eighth International Symposium on Information and Communication Technology, pp. 197-203. https://doi.org/10.1145/3155133.3155180 